\title{
P-S Time Observation based on Laser Interferometer Seismic Signal Data
}

\author{
Minwoo Lim, Kyunghyun Lee, Jungkeun Oh and Kwanho You \\ Sungkyunkwan University, Suwon, 440-746, Korea \\ didwktlr@skku.edu,naman2001@skku.edu,ojkorea@skku.edu,khyou@skku.edu
}

\begin{abstract}
In seismic signal detection, it is important to detect seismic wave and P-S wave arrival time. Seismometers or accelerometers which are used as seismic wave measurement system have some limits to detect seismic signal. In order to overcome these limits, we propose the seismic wave measurement device based on a heterodyne laser interferometer. Heterodyne laser interferometer is used as the distance measurement device which has measurable range from nanometer to several meters. Therefore we model seismic wave detection system based on heterodyne laser interferometer to measure $P$-S time for epicenter. Finally, we use STA/LTA algorithm to detect P-S time accurately. It is shown that heterodyne laser interferometer can be used as seismic detection device.
\end{abstract}

Keywords: Heterodyne Laser Interferometer, STA/LTA Algorithm, P-S Time, Seismic Signal

\section{Introduction}

Nowadays, there are many natural disasters such as typhoon, earthquake, tsunami, etc. Earthquake happens suddenly and moves fast. In order to prepare for the earthquake, we need for fast and accurate seismic signal detection. The mainly used devices to measure seismic wave are seismometer or accelerometer [1-2]. These apparatuses consist of electronic sensors which are based on piezo-electricity and piezo-resistive effect. However, there exist limitations of low resolution and narrow bandwidths.

In order to solve this problem, Gardner [3] proposed a seismometer based on a fiberoptic interferometry which has a high sensitivity. Wu [4] proposed Fiber Bragg Grating (FBG) based accelerometer which is robust to EMI, and has a signal multiplexing capability. Gevorgyan [5] suggested the single-layer open-flat-coil (OFC) oscillatorbased sensitive platform technology to overcome the defect of seismometer and accelerometer. Acernese [6] described a new low-frequency seismic sensor by using Michelson interferometer. Loh [7] proposed the interferometric accelerometer which consists of a suspended bulk-micromachined proof mass with protruding fingers. Araya [8] suggested the highly sensitive wideband seismometer based on the laser interferometer.

In this paper, we propose a seismic signal detection method based on a laser interferometer system. Laser interferometer is a displacement measuring device. Using laser Doppler effect, laser interferometer measures distance. It is used for precision length measurement applied in the fields of semiconductor manufacture and robotics. Moreover, it covers a wide displacement range, from sub-nanometer (nm) to several meter scale. This paper is written as follows: In Section 2, we analyze a laser interferometry system. Section 3 explains an STA/LTA algorithm, P-wave and S-wave. 
The performance is demonstrated by a simulation in Section 4. The conclusion appears in Section 5.

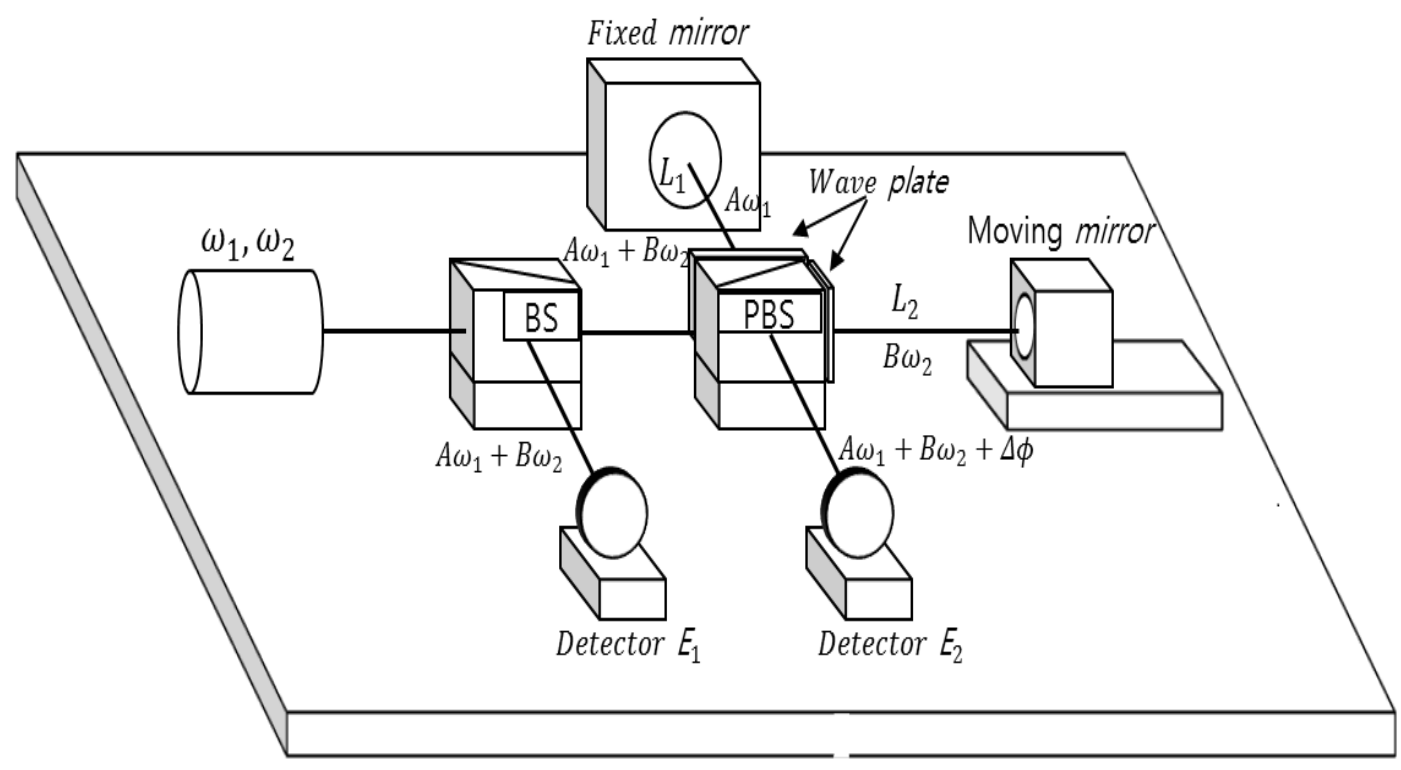

Figure 1. Heterodyne laser interferometer

\section{Heterodyne Laser Interferometer}

Heterodyne laser interferometer emits laser light source which consists of two orthogonal beams with difference frequencies [9-10]. The laser light is divided by the beam splitter (BS) into two beams. One is the reference signal and the other is the measurement signal. The laser lights are denoted by $A \omega_{1}+B \omega_{2}$. The reference beam is collected by a photo detector $A$. The intensity of reference electric fields from detector $A$ can be expressed as follows

$$
\begin{aligned}
& E_{A 1}=\frac{1}{\sqrt{2}} A e^{j\left(\omega_{1} t+\phi_{A}\right)} \\
& E_{A 2}=\frac{1}{\sqrt{2}} B e^{j\left(\omega_{2} t+\phi_{B}\right)}
\end{aligned}
$$

The output signal intensity $I_{r}$ measured by photo detector $A$ is

$$
\begin{aligned}
I_{r} & \propto\left(E_{A 1}+E_{A 2}\right)\left(E_{A 1}+E_{A 2}\right)^{*} \\
& =\frac{1}{2}\left(A^{2}+B^{2}\right)+A B \cos \left[\Delta w t+\left(\phi_{B}-\phi_{A}\right)\right]
\end{aligned}
$$

The measurement beam is divided by a polarizing beam splitter (PBS) as $A \omega_{1}$ and $B \omega_{2}$. One signal $\left(A \omega_{1}\right)$ is reflected by a fixed mirror. The other signal $\left(B \omega_{2}\right)$ is reflected by a moving mirror. These signals are collected by a photo detector $B$. With no additive error, the intensity of measurement beam is represented as follows. 


$$
\begin{aligned}
& E_{B 1}=\frac{1}{\sqrt{2}} A e^{j\left(\omega_{1} t+\phi_{A}\right)} \\
& E_{B 2}=\frac{1}{\sqrt{2}} B e^{j\left(\omega_{2} t+\phi_{B}+\Delta \phi\right)}
\end{aligned}
$$

The output signal collected by a photo detector $B$ is proportional to wave intensity.

$$
\begin{aligned}
I_{m} & \propto\left(E_{B 1}+E_{B 2}\right)\left(E_{B 1}+E_{B 2}\right)^{*} \\
& =\frac{1}{2}\left(A^{2}+B^{2}\right)+A B \cos \left[\Delta w t+\left(\phi_{B}-\phi_{A}\right)+\Delta \phi\right]
\end{aligned}
$$

where $\Delta \omega$ is defined as $\Delta \omega=\omega_{2}-\omega_{1} . \Delta \phi$ is the frequency difference caused by Doppler effect. Using a high pass filter, we eliminate DC components. Then, the AC components of $I_{r}$ and $I_{m}$ remain as follows.

$$
\begin{aligned}
& I_{r, a c} \propto A B \cos (\Delta \omega t), \\
& I_{m, a c} \propto A B \cos (\Delta \omega t+\Delta \phi)
\end{aligned}
$$

To measure the phase in high accuracy, we use a lock-in amplifier to obtain phase information. $I_{r, a c}$ and $I_{m, a c}$ signals are calculated through a multiplier. Signal $I_{r}$ is divided into two signals. One has the same phase with $I_{r}$ and the other is 90 degree shifted $I_{r}$. Each signal is multiplied by $I_{m}$. We can obtain phase information which is needed to measure length. Moreover, the multiplied results pass through a low-pass filter. Then, they are represented in sine and cosine waveforms.

$$
\begin{aligned}
& I_{x} \propto \frac{A B}{2} \cos \Delta \phi, \\
& I_{y} \propto \frac{A B}{2} \sin \Delta \phi
\end{aligned}
$$

We can obtain $\Delta \phi$ as follows,

$$
\Delta \phi=\tan ^{-1}\left(\frac{I_{y}}{I_{x}}\right)
$$

Finally, we can determine the difference in the optical length $(\Delta L)$ by using the equation of $\Delta L=\Delta \phi \lambda / 4 \pi n$. $\lambda$ is the mean of wavelength, $n$ is a refractive index, and $\Delta L$ is the difference of length between a fix mirror and a moving mirror.

The intensity signals of $I_{x}$ and $I_{y}$ is expressed as sine and cosine functions in Eq. (6). In an ideal circumstance, Lissajous' figure forms a circle in the $I_{x}-I_{y}$ plane. The trajectory of intensity according to the change of phase is plotted in Figure 2. 


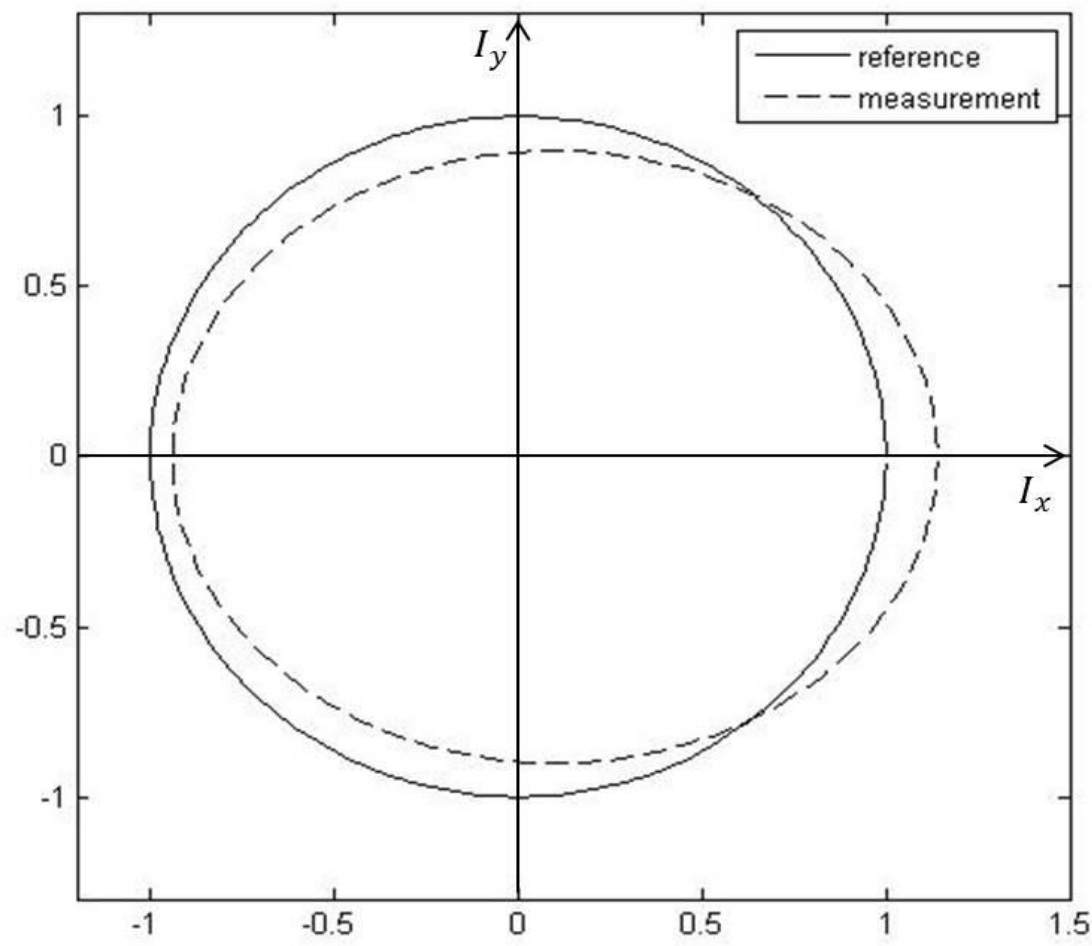

Figure 2. $I_{x}-I_{y}$ plane

\section{STA/LTA Algorithm for P-S Time Measurement}

Generally, seismic waves consist of $\mathrm{P}$-wave, S-wave and surface wave. $\mathrm{P}$-wave is a longitudinal wave and arrives first. S-wave is a transverse wave and arrives secondly. Surface wave moves along surface and is the last to arrive. By using the difference of P-wave and Swave arrival we can measure the distance of epicenter. Therefore, we use the STA/LTA algorithm to calculate P-S time [11]. STA/LTA algorithm uses two moving time windows. To calculate the STA/LTA algorithm, the absolute amplitude of each seismic signal data is used. Next, the average of absolute amplitude is calculated by each window size. By using the average of absolute amplitude, we can obtain a value defined as STA/LTA ratio. In STA/LTA algorithm, we need to determine several factors such as STA window duration, LTA window duration, STA/LTA trigger threshold level, and STA/LTA detrigger threshold level. Moreover, to optimize triggering of seismic recorder, we set up these parameters which are trigger filters, pre-event time (PEM), post-event time (PET), and trigger voting scheme.

The STA/LTA algorithm which is a standard trigger method in seismic recorders measures the ratio of energy density between short-term and long-term [12-14]. This algorithm estimates a seismic signal in two moving time window. If the amplitude of a seismic signal is defined as $a(t)$, the short-term average is represented as 


$$
\operatorname{STA}(\tau)=\frac{1}{n_{s}} \sum_{t=\tau-n_{s}}^{\tau} a(t)
$$

where $n_{s}$ is the length of time window for short-term average. Similarly, the long-term average is represented as

$$
\operatorname{LTA}(\tau)=\frac{1}{n_{l}} \sum_{t=\tau-n_{l}}^{\tau} a(t)
$$

where $n_{l}$ is the length of time window for long-term average

The short-term average measures the instant amplitude of the seismic signal. While the long-term average calculates the real time average of seismic wave amplitude. Therefore it is less sensitive to a slow increase in energy. The STA/LTA algorithm measures the signal-tonoise ratio. The time when the STA/LTA ratio is over the user-defined threshold value is used to calculate the P-S time.

\section{Simulation}

In this section, we demonstrate the application of seismic signal measurement based on a laser interferometer and apply STA/LTA algorithm to measure the P-S time. Figure 3 presents the seismic signal detection with laser interferometer.

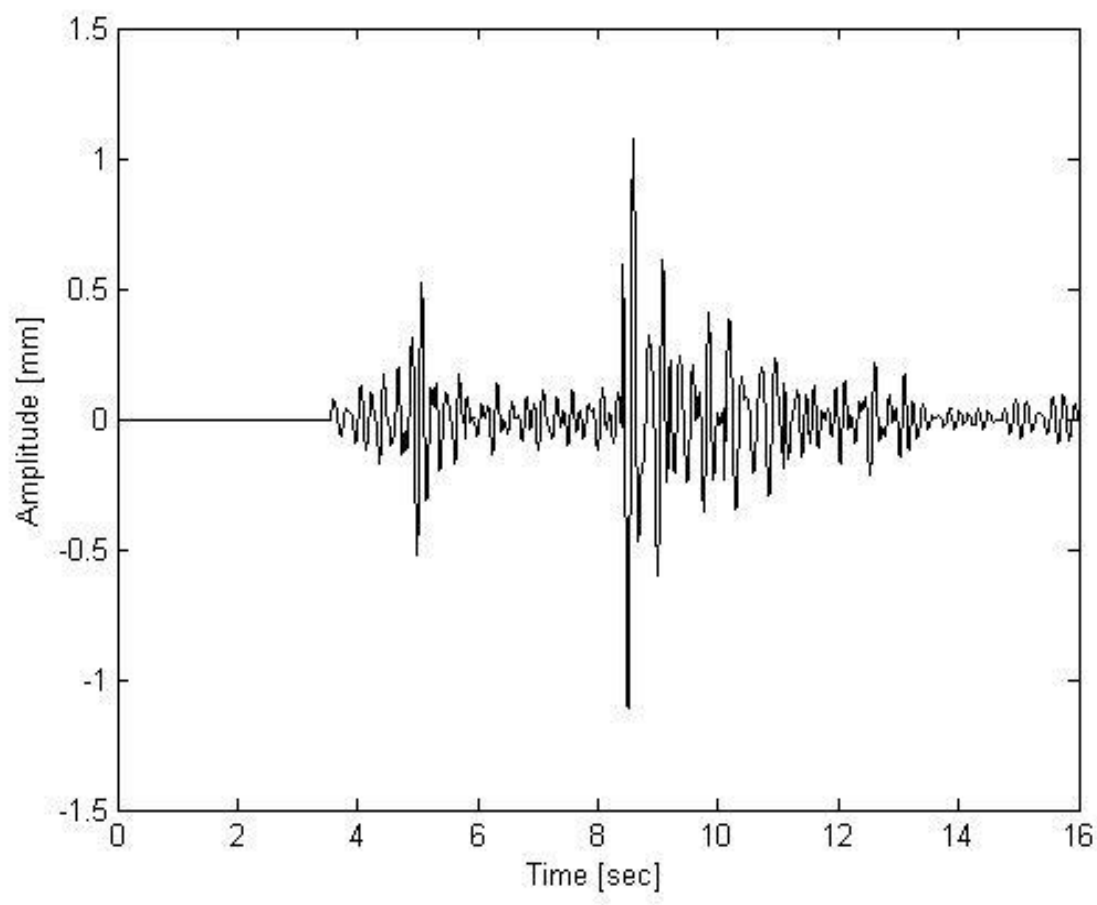

Figure 3. Seismic signal measured by laser interferometer 
Wavelength $(\lambda)$ is set as $632.991 \mathrm{~nm}$ and refractive index $(n)$ is 1.00000002665 . By using the laser interferometer equation, we can measure the seismic wave movement.

Figure 4 shows the application of STA/LTA algorithm in simulated data to set STA window size of 400 . We set up the window length of LTA as 10 times of STA and the threshold value as 2 times of average of STA/LTA. When STA/LTA value is over the threshold value, we can detect the $\mathrm{P}$-wave arrival time and $\mathrm{S}$-wave arrival time, respectively. $\mathrm{P}$-and S-wave arrival time are measured as $3.6 \mathrm{~s}$ and $8.4 \mathrm{~s}$. If the velocity of P-wave is $8 \mathrm{~km} / \mathrm{s}$ and the velocity of $S$ wave is $4 \mathrm{~km} / \mathrm{s}$, we can determine that epicentral distance is $38.4 \mathrm{~km}$ by using the equation of $D=\left(V_{P} V_{S} / V_{P}-V_{S}\right) T_{P S} . D$ is epicentral distance. $V_{P}$ is P-wave velocity. $V_{S}$ is $\mathrm{S}$-wave velocity $T_{P S}$ is $\mathrm{P}-\mathrm{S}$ time.

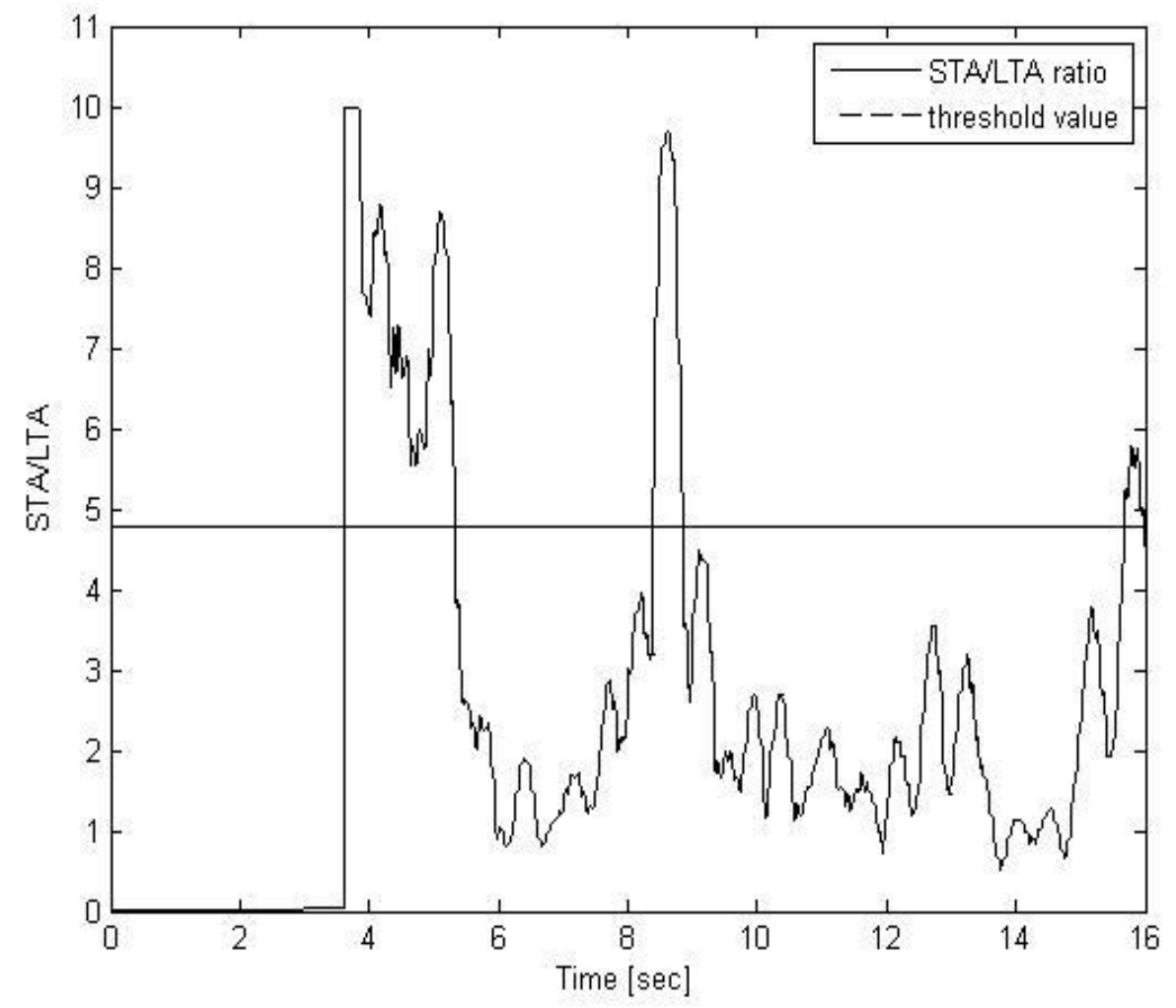

Figure 4. STA/LTA ratio with window size of 400

Figure 5 shows the application of STA/LTA algorithm in simulated data to set STA window size as 300. LTA window size is the same size as in Figure 4. The threshold value sets two times of STA/LTA ratio. We measure the P-wave arrival and S-wave arrival time again. Each P-S arrival time was measured as 3.6s and 8.4s, respectively. We assumed the same velocity of P-wave and S-wave in Figure 4. Then, we can determine the epicentral distance. The result of the epicentral distance with a window size of 300 is almost equal to that of a window size of 400 . However, STA value with a small window size is more sensitive. Therefore, it shows more fluctuations according to various STA/LTA ratio and has more occurrences when STA/LTA ratio is above the threshold value. It shows a steep slope at Pwave arrival time and S-wave arrival time. 


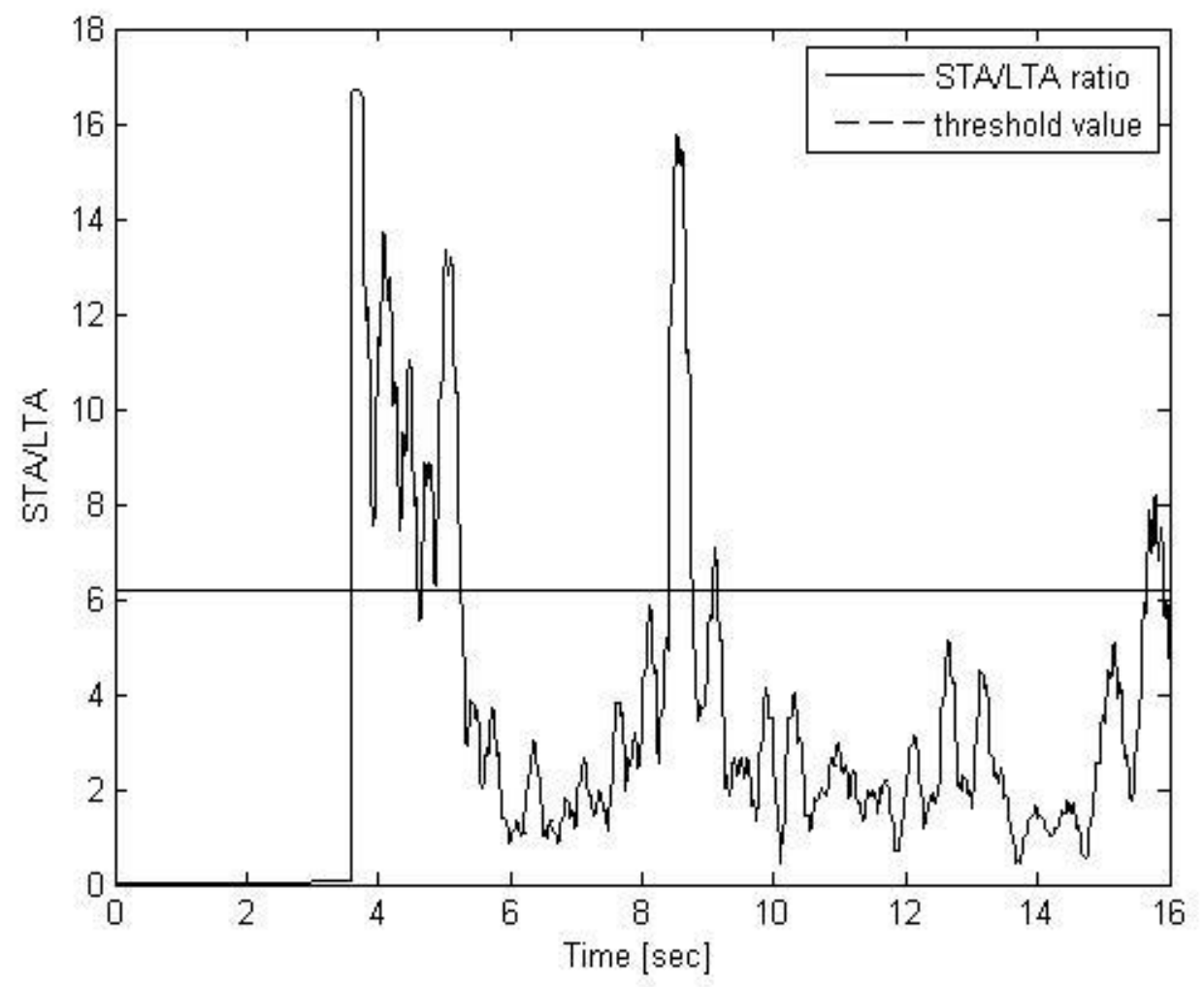

Figure 5. STA/LTA ratio with window size of 300

\section{Conclusions}

In this paper, we purpose a seismic signal measurement method by using a heterodyne laser interferometer. Laser interferometer can measure the length using its intensity signal. By using a laser interferometer, we can detect the seismic wave in length scale and enhance the measurement accuracy in seismic signal detection. In order to prove the proposed P-S time detection algorithm, we set up the system modeling. Moreover, we use STA/LTA algorithm to measure P-S time. We confirm that laser interferometer can measure seismic signal as a seismometer.

\section{Acknowledgements}

This research was supported by Basic Science Research pro-gram through the National Research Foundation of Korea (NRF) funded by the Ministry of Education, Science and Technology (2013R1A1A2006728).

\section{References}

[1] F. Acernese, R. D. Rosa, G. Giordano, R. Roman and F. Barone, "Mechanical monolithic accelerometer for suspension inertial damping and low frequency seismic noise measurement", Journal of Physics: Conference Series, vol. 122, (2008).

[2] F. Garcia, L. Hixson, H. Elmer, C. Orozco and I. Horacio, "Seismic Accelerometer", IEEE Instrumentation and Measurement Technology Conference, vol. 3, (1999), pp. 1342-1346. 
[3] D. L. Gardner, T. Hofler, S. R. Baker, S. L. Yarber and S. L. Garrett, "A fiber-optic interferometric seismometer", Journal Lightwave Technology, vol. 5, (1987) July, pp. 952-959.

[4] J. Wu, V. Masek and M. Cada, "The possible use of fiber bragg grating based accelerometers for seismic measurements", Canadian Conference Electrical and Computer Engineering, (2009) July, pp. 860-863.

[5] S. G. Gevorgyan, V. S. Gevorgyan, H. G. Shirinyan, G. H. Karapetyan and A. G. Sarkisyan, "A radically new principle of operation seismic detector of nano-scale vibrations", IEEE Transactions on Applied Superconductivity, vol. 17, (2007) June, pp. 629-632.

[6] F. Acernese, R. D. Rosa, F. Garufi, R. Roman and F. Barone, "A Michelson interferometer for seismic wave measurement", Theoretical analysis and system performances", Proceeding of SPIE-The international Society for Optical Engineering, vol. 6366, (2006) September 13-14; Stockholm, Sweden.

[7] N. C. Loh, M. A. Schmidt and S. R. Manalis, "Sub-10 $\mathrm{cm}^{3}$ interferometric accelerometer with nano-g resolution", Journal Microelectromechanical Systems, vol. 11, (2002) June, pp. 182-187.

[8] A. Araya, K. Kawabe, T. Sato, N. Mio and K. Tsubono, "Highly sensitive wideband seismometer using a laser interferometer", Review of Scientific Instrument, vol. 64, (1993) May, pp. 1337-1341.

[9] C. M. Wu and C. S. Su, "Nonlinearity in measurements of length by optical interferometry", Measurement Science and Technology, vol. 7, (1996) pp. 62-68.

[10] W. Lee, J. Lee and K. You, "Improved measurement accuracy in heterodyne laser interferometer using WDF", Electronics Letter, vol. 45, (2009) October, pp. 1085-1087.

[11] M. Withers, R. Aster, C. Young, J. Beiriger, M. Harris, S. Moore and J. Trujillo, "A comparison of select trigger algorithms for automated global seismic phase and event detection", Bulletin of Seismological Society of America, vol. 88, (1998) February, pp. 95-106.

[12] A. A. Velasco, S. Hernandez, T. Parsons and K. Pankow, "Global ubiquity of dynamic earthquake triggering", Nature Geoscience, vol. 1, (2008) May, pp. 375-379.

[13] R. Sleeman and T. V. Eck, "Robust automatic P-phase picking: An on-line implementation in the analysis of broadband seismogram recordings", Physics of Earth Planetary Interiors, vol. 113, (1999) June, pp. 265-275.

[14] B. K. Sharma, A. Kumar and V. M. Murthy, "Evaluation of Seismic Events Detection Algorithms", Journal Geological Society of India, vol. 75, (2010) March, pp. 553-538.

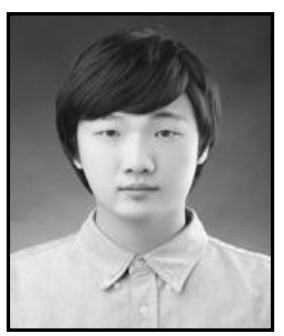

\section{Minwoo Lim}

He received his B.S. degree in electrical engineering from Sungkyunkwan University in 2013. He is working as a research staff in applied optimization lab. in Sungkyunkwan University. His research interests are heterodyne laser interferometer error compensation, seismic wave detection with laser interferometer, and sliding mode control.

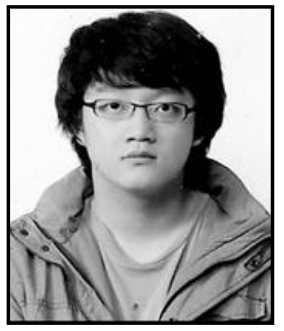

\section{Kyunghyun Lee}

$\mathrm{He}$ received his B.S. degree in electrical engineering from Sungkyunkwan University in 2013. He is working as a research staff in applied optimization lab. in Sungkyunkwan University. His current interest fields are intelligent control, geolocation problem with TDOA/FDOA, measurement sensor development, estimation theory and real-time nonlinear systems. 


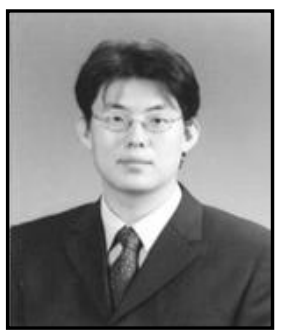

\section{Jungkeun Oh}

He received his B.S. degree in electrical engineering from Hallym University in 2002, and received his M.S. degree from Sungkyunkwan University in 2005 . He is working as a research staff in applied optimization lab. in Sungkyunkwan University. His current interest fields are radio resource management, geolocation problem with TDOA/FDOA, measurement sensor development, and real-time nonlinear systems.

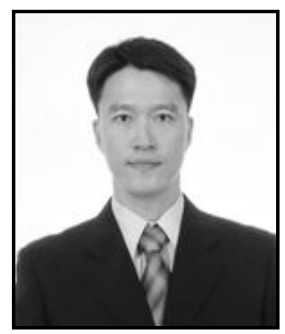

\section{Kwanho You}

He received his B.S. and M.S. degrees in electrical engineering from Korea Advanced Institute of Science and Technology (KAIST) in 1993 and 1996, and received his Ph.D. Degree from the University of Minnesota in 2000, respectively. He had worked as a faculty at Texas A\&M University-Kingsville. In 2001, he joined the School of Information and Communication Engineering at Sungkyunkwan University. His research interests are in nonlinear and optimal control, radio resource management in wireless communication, adaptive optimization methods in nonlinear process, and estimation theory. 
International Journal of Control and Automation Vol.7, No.4 (2014) 